\title{
MEDIACIÓN SOCIAL Y CULTURA DE PAZ
}

\author{
SOCIAL MEDIATION AND CULTURE OF PEACE
}

\author{
Francisca Silva-Hernández ${ }^{1} *$ (iD \\ 1. Universidad Juárez Autónoma de Tabasco, México. fany987@hotmail.com \\ *Correspondencia del Autor: Francisca Silva-Hernández, correo electrónico: fany987@hotmail.com.
}

\section{RESUMEN}

Las relaciones sociales en una sociedad conllevan un proceso estructural y dinámico, en el que la interacción crea situaciones de convivencia, pero también de diferencias, conflicto o violencia entre las personas. La comunidad además de ser un espacio geográfico configura elementos sociales, culturales, intereses propios y compartidos, para comprender, atender y mitigar situaciones de índole conflictiva en la comunidad, la mediación social representa un mecanismo que coadyuva a un proceso de cultura de paz, a partir, de integrar y desarrollar aspectos base del mecanismo citado, lo que implica desarrollar o aumentar en los miembros de la comunidad estrategias de diálogo, comunicación y participación en toma de decisiones así como gestionar y resolver conflictos. Este documento identifica elementos base de la mediación social asociado a la cultura de paz, para contemplar el ejercicio de diálogo y comunicación entre los miembros de una comunidad, creando escenarios progresivos de pluralidad y bienestar.

Palabras clave: comunidad; diálogo; prevención.

Cómo citar:

Silva-Hernández, Francisca. (2021). MEDIACIÓN SOCIAL Y CULTURA DE PAZ. Revista de Investigaciones Universidad del Quindio, 33(1), 241-244. https://doi.org/10.33975/riuq.vol33n1.591 


\begin{abstract}
Social relations in society involve a structural and dynamic process, in which interaction creates situations of coexistence, but also of differences, conflict or violence between people. The community, in addition to being a geographical space, configures social, cultural elements, own and shared interests, to understand, attend and mitigate situations of a conflictive nature in the community, social mediation represents a mechanism that contributes to a process of the culture of peace, starting from, integrating and developing basic aspects of the aforementioned mechanism, which implies developing or increasing in the members of the community strategies of dialogue, communication and participation in decision-making as well as managing and resolving conflicts. This document identifies basic elements of social mediation associated with the culture of peace, to contemplate the exercise of dialogue and communication between the members of a community, creating progressive scenarios of plurality and well-being.
\end{abstract}

Keywords: community; dialogue; prevention.

\section{INTRODUCCIÓN}

Los espacios de interacción social como lo es una comunidad crean condiciones de convivencia en el que emergen un sinfín de relaciones sociales con intereses, necesidades, creencias y valores comunes y compartidas que otorgan unidad e integridad a cada miembro y en conjunto.

Las condiciones de convivencia están inmersas en espacios representativos, así como mediante un lenguaje simbólico que caracteriza y da identidad a la comunidad. En ese escenario de convivencia emergen diferencias a partir de la diversidad de pensamiento y conductas, lo que puede suscitar conflictos.

Para ello, la justicia alternativa a través de los mecanismos alternativos de solución de controversias, representan una vía de avance para atender y coadyuvar el diálogo y comunicación (De Moragas, 2007) en miembros de una comunidad. Tal es el caso de la mediación social, la cual refleja características y elementos que constituye pluralidad, consenso, atención de intereses y necesidades de las personas involucradas en conflictos, previendo aspectos de la comunidad y potencializando aspectos culturales y sociales de la misma.
Este documento estructura la relación de mediación social y cultura de paz, en pro de una educación formal e informal adoptada y reproducida por los miembros de la sociedad en diversos escenarios, configurando así una calidad de vida para los integrantes de la misma.

\section{METODOLOGÍA}

Los procesos complejos de comunicación en una comunidad dirigen a un estado dinámico entre las personas. La comprensión de conflictos en dicha dinámica sobrelleva a considerar elementos estructurales de la agrupación y su organización.

En ese proceso de comprensión, se describen aspectos de la mediación social y cultura de paz, proveniente de una investigación cualitativa con revisión documental de interés concerniente a las realidades sociales.

\section{RESULTADOS}

\section{Mediación social}

Este mecanismo está previsto para resolver conflicto grupal, comunitario, con el fin de consensuar ideas, diálogo, previendo aspectos socio histórico e identidad de la comunidad. 
De acuerdo a Martín Serrano, la teoría de la mediación social ofrece un nuevo objeto para las ciencias sociales: el estudio de la producción, transmisión y utilización de la cultura, a partir del análisis de los modelos culturales y de sus funciones (Martín, 1985). Visualiza lo socio histórico a partir de elementos que describen aspectos culturales de la comunidad o grupo.

Representa una singularidad de intervención de terceras partes, en y sobre contextos sociales de multiculturalidad significativa, orientada hacia la consecución del reconocimiento del otro y el acercamiento de las partes, la comunicación y comprensión mutua, el aprendizaje y desarrollo de la convivencia, la regulación de conflictos y la adecuación institucional, entre actores sociales o institucionales etnoculturalmente diferenciados (Delgado, 2011). Versa el proceso de la mediación a partir del reconocimiento y empatía de la otra parte involucrada, permitiendo procesos de adaptación o regulación en conductas y consenso, asimismo, es aplicable a paradigmas de transformaciones de las sociedades y de las personas, que se anticipan y acompañan a esos cambios (Martín, 1976).

Castro (2018) refiere que la intervención pretende transformar, participar, reconciliar un problema de riesgo social particular, que cubra las necesidades humanas para un desarrollo integral, sostenible, mejorando la calidad de vida.

La mediación social implica comprender particularidades del grupo o comunidad que permitan revalidar esas mismas particularidades, afín de mejorar las condiciones de convivencia entre los miembros y futuras generaciones.

\section{Cultura de paz}

Con base a Naciones Unidas, cultura de paz es conjunto de valores, actitudes, tradiciones, comportamientos y estilos de vida basados en: respeto a la vida, fin de violencia, diálogo, cooperación, respeto pleno de los principios de soberanía, integridad territorial e independencia política, respeto de los derechos humanos y libertades fundamentales, arreglo pacífico de conflictos, desarrollo sustentable y sostenible, respeto al derecho al desarrollo, igualdad entre hombres y mujeres, libertad de expresión, opinión e información, adhesión a los principios de libertad, justicia, democracia, tolerancia, solidaridad, cooperación, pluralismo, diversidad cultural, diálogo y entendimiento de la sociedad y naciones, mismos que deben favorecer la paz (Naciones Unidas, 1999).

De lo anterior, se procura equilibrar o tener un balance de condiciones propicias de respeto, tolerancia, solidaridad y cooperación entre miembros de una comunidad, por lo que hay que prever que en conflictos que emergen en la misma se debe de tener una intervención que promueva la participación activa de los actores mediante acciones con capacidad de toma de decisión, protagonismo en gestionar y resolver conflictos, respeto a la pluralidad, desarrollar aspectos democráticos (Izquierdo, 2007) y consensuados.

En este proceso de cultura de paz, las personas inmersas en una comunidad se vuelven autogestoras de su conflicto en caso tal, aprovechar los espacios físicos, digitales (parque, iglesias, delegación, redes sociales, entre otros) con los que cuenta la comunidad para la asociación integral de las relaciones entre los miembros con valores, creencias y otros afines.

A partir de estas vías de comunicación se generan transformaciones en el funcionamiento de la sociedad [producción y reproducción social] (Romo, 2011), en el que el mensaje de la comunicación ubica en tiempo y espacio a sujetos capaces de comprender situaciones de interés individual y colectivo.

La paz como un hecho social, es un valor que orienta el desarrollo de la cultura (Hernández et al., 2017) por lo que desde su origen multifactorial requiere una comprensión multidisciplinar con el objeto de saber las percepciones e ideas y así proceder a cambios de asumir compromisos y 
construir nuevas normalidades de conductas que alienten a una cultura de paz.

Es así que la mediación social permite a partir de su análisis socio histórico regular las funciones de la comunidad o grupo contribuyendo a identificar aspectos a mejorar, evitar o modificar, en pro de un estado de bienestar, democrático, bien común.

\section{DISCUSIÓN}

En el proceso de mediación social el mediador apoya a los involucrados a llegar a un acuerdo positivo, eficaz, mejorando así la convivencia ante contextos religiosos, culturales, desigualdad social, género, discriminación y todo escenario de configuración de nuevas estructuras familiares.

La asociación de mediación social y cultura de paz, crean un proceso de educación formal e informal en el que los sujetos de diversas edades propician en su contexto el desarrollo de las habilidades de este mecanismo, fortaleciendo relaciones participativas, flexibilidad en toma de decisiones que orientan a objetivos concretos. El afianzar las relaciones sociales apoyan a la prevención de conflictos y una sana convivencia asegurando el respeto hacia el otro y en garantía de todo derecho humano.

\section{REFERENCIAS}

1. Castro Clemente, Clemente. (2018). Intervención y mediación social. Definición y contextos profesionales. Aldaba, (42), 51-62. https://doi.org/10.5944/aldaba.42.2017.20805

2. De Moragas Spá, Miquel. (2007). La mediación social y los enfoques de la teoría de la comunicación. Mediaciones sociales, (1), 261-269.

3. Delgado Aguilar, Ana. (2011). La mediación social e intercultural en espacios públicos. Investigación curso en mediación social e intercultural, Actas del I Congreso Internacional sobre Migraciones en Andalucia, 1055-1064.

4. Hernández Arteaga, Isabel; Luna Hernández, José Alberto y Cadena Chala, Martha Cecilia. (2017). Cultura de paz: una construcción desde la educación. Revista Historia de la educación latinoamericana, 19(28), 149-172. https://doi.org/10.19053/01227238.5596

5. Izquierdo Muciño, Martha Elba. (2007). Por una cultura de paz. Revista Espiral, XIV (40), 157175.

6. Martín Serrano, Manuel. (1985). "La mediación de los medios de comunicación”, en MORAGAS, M. "Sociología de la comunicación de masas", Barcelona: Gustavo Gili.

7. Martín Serrano, Manuel. (1976). Diccionario de Ciencias Sociales. Madrid: Instituto de Estudios Políticos.

8. Naciones Unidas. (1999). 53/243. Declaración y Programa de Acción sobre una Cultura de Paz. Declaración sobre una cultura de paz.

9. Romo Franco, Daniel. (2011). Reseña de "La mediación social” de Manuel Martin Serrano. Razón y palabra (75). 